\title{
Local Symplectic Invariants for Curves
}

\author{
Niky Kamran ${ }^{1}$, Peter Olver ${ }^{2}$ and Keti Tenenblat ${ }^{3}$ \\ 1 Department of Mathematics and Statistics, McGill University, \\ Montreal, QC, H3A 2K6, Canada. \\ 2 School of Mathematics, University of Minnesota, \\ Minneapolis, MN, 55455, USA. \\ 3 Departamento de Matemática, Universidade de Brasília, \\ Brasília 70910 DF, Brazil.
}

April 3, 2009

\section{Introduction.}

To the best of our knowledge, the study of the local symplectic invariants of submanifolds of Euclidean space was initiated by Chern and Wang in 1947, [6]. They considered mainly the case of curves and hypersurfaces, and obtained structure equations defining a set of local symplectic differential invariants for these objects. We should explain at this stage that by "symplectic invariants" we mean invariants under the direct product of the affine linear symplectic group of $\mathbb{R}^{2 n}$ endowed with the standard symplectic form with the infinite-dimensional pseudo-group of reparametrizations of the submanifolds. This is in contrast with the case in which one considers the full infinite-dimensional symplectomorphism group of the ambient $\mathbb{R}^{2 n}$. Indeed, in the latter case, the theorem of Darboux implies that submanifolds have no local differential invariants. (On the other hand, Ekeland and Hofer's discovery of symplectic capacity, [7], shows that there are nontrivial global invariants. These lie beyond the scope of this work.). The case we are interested in is much closer in spirit to ordinary Euclidean or affine 
differential geometry, where the ambient space is a homogeneous space for the action of a finite-dimensional Lie group, and where submanifolds enjoy a wealth of local differential invariants.

Our purpose in this paper is to further develop the line of research initiated in [6] by constructing explicitly a complete set of local symplectic invariants for curves, through two different approaches. The first approach is to work "from the bottom up" by successively differentiating the tangent vector to the curve and using the non-degenerate inner product associated to the symplectic form to construct a symplectic Frenet frame. The structure functions of the frame are then the local invariants of curves. We will sometimes refer to these local differential invariants as the symplectic curvatures of the curve. The other approach is to work "down from the top" by constructing explicitly the invariants by the method of moving frames, [5], using the algorithm given in [8]. See also $[9,12]$ for further developments and applications of the equivariant approach to moving frames. We show explicitly that these approaches lead to the same Frenet frame and to the same same set of local differential invariants. We also investigate the case of curves in $\mathbb{R}^{4}$ having the property that all their differential invariants are constant. By a general theorem of Cartan, [9], we know that this is the case if and only if the curve is the orbit of a one-parameter subgroup of the affine symplectic group. We give a classification of these curves according to the algebraic type of the spectrum of their corresponding Frenet matrices.

We would like to mention that there has been some recent work related to the symplectic geometry of submanifolds of Euclidean space including [1], [3] and [13]. However, [1] deals with the specific case of fanning curves in a symplectic manifold, while [13] constructs differential invariants for two other actions of the affine symplectic group, which are thus different from ours. Moreover, our approach yields not only the invariants, but also a Frenet frame, obtained 
in analogy with the classical constructions from Euclidean geometry, and the method of moving frames. This Frenet frame is indispensible when trying to reconstruct the parametrized curves corresponding to a given choice of symplectic invariants. Similar remarks apply to [3], where the authors consider the problem of computing the differential invariants for different linear actions of the symplectic group, corresponding to different irreducible representations of the symplectic group. However, the explicit formulas for the differential invariants are only obtained for the lowest dimensional symplectic groups.

Our paper is organized as follows. After reviewing in Section 2 some basic definitions from symplectic geometry, we introduce in Section 3 the concepts of symplectic arc length and symplectic regular curve, which takes care of the reparametrization freedom for the curves under consideration. Section 4 is devoted to the construction by successive differentiations of the symplectic Frenet frame and the corresponding local differential invariants. We then prove an existence and uniqueness theorem for curves with prescribed symplectic curvatures, analogous to the corresponding theorem in Euclidean geometry. In Section 5, we show by explicit computation that the same Frenet frame and local differential invariants can be constructed by the method of moving frames, using the algorithm described in [8]. Section 7 is devoted to a general discussion of the case of curves in $\mathbb{R}^{4}$ of constant symplectic curvatures. An algebraic classification of the spectrum of the Frenet matrix is given in terms of conditions on the numerical values of the constant symplectic curvatures. 


\section{Preliminaries.}

We consider $M=\mathbb{R}^{2 n}$ endowed with the standard symplectic form $\Omega$ given in global Darboux coordinates by

$$
\Omega=\sum_{i=1}^{n} d x^{i} \wedge d y^{i}
$$

Each tangent space is thus endowed with the symplectic inner product ${ }^{1}$ defined for $\mathbf{u}=\left(x^{1}, \ldots, x^{n}, y^{1}, \ldots, y^{n}\right)$ and $\mathbf{v}=\left(\xi^{1}, \ldots, \xi^{n}, \omega^{1}, \ldots, \omega^{n}\right)$ written in the canonical basis by

$$
\begin{aligned}
\langle\mathbf{u} ; \mathbf{v}\rangle & =\Omega(\mathbf{u}, \mathbf{v})=\mathbf{u}^{T} J \mathbf{v} \\
& =\sum_{i=1}^{n}\left(x^{i} \omega^{i}-y^{i} \xi^{i}\right), \quad \text { where } \quad J=\left(\begin{array}{cc}
0 & I_{n} \\
-I_{n} & 0
\end{array}\right) .
\end{aligned}
$$

The symplectic group $\operatorname{Sp}(2 n, \mathbb{R})$ is the subgroup of $\mathrm{GL}(2 n, \mathbb{R})$ which preserves the symplectic inner product. It is of dimension $n(2 n+1)$. The Lie algebra $\mathfrak{s p}(2 n, \mathbb{R})$ of $\operatorname{Sp}(2 n, \mathbb{R})$ is the vector space of all matrices of the form

$$
X=\left(\begin{array}{cc}
U & V \\
W & -U^{t}
\end{array}\right)
$$

where $U, V$ and $W$ are $n \times n$ matrices satisfying

$$
W=W^{t}, \quad V=V^{t},
$$

and where the Lie bracket is given by the usual matrix commutator. We will refer to the semi-direct product $G=\operatorname{Sp}(2 n, \mathbb{R}) \ltimes \mathbf{R}^{2 n}$ of the symplectic group by the translations as the group of rigid symplectic motions. A rigid symplectic

\footnotetext{
${ }^{1} \mathrm{By}$ definition, a symplectic inner product is a nondegenerate, skew-symmetric bilinear form on the underlying vector space. There is, obviously, no requirement of positive definiteness.
} 
motion $(A, \mathbf{b}) \in G$ is thus the same thing as an affine symplectic transformation, acting on points $\mathbf{z} \in \mathbf{R}^{2 n}$ via

$$
\mathbf{z} \longmapsto A \mathbf{z}+\mathbf{b}
$$

A symplectic frame is by definition a smooth section of the bundle of linear frames over $\mathbb{R}^{2 n}$ which assigns to every point $\mathbf{z} \in \mathbb{R}^{2 n}$ an ordered basis of tangent vectors $\left\{\mathbf{a}_{1}, \ldots, \mathbf{a}_{2 n}\right\}$ such that

$$
\begin{aligned}
\left\langle\mathbf{a}_{i} ; \mathbf{a}_{j}\right\rangle=\left\langle\mathbf{a}_{i+n} ; \mathbf{a}_{j+n}\right\rangle & =0, & & 1 \leq i, j \leq n, \\
\left\langle\mathbf{a}_{i} ; \mathbf{a}_{j+n}\right\rangle & =0, & & 1 \leq i \neq j \leq n, \\
\left\langle\mathbf{a}_{i} ; \mathbf{a}_{i+n}\right\rangle & =1, & & 1 \leq i \leq n .
\end{aligned}
$$

The structure equations for a symplectic frame are thus of the form

$$
\begin{aligned}
d \mathbf{a}_{i} & =\sum_{k=1}^{n} \omega_{i k} \mathbf{a}_{k}+\sum_{k=1}^{n} \theta_{i k} \mathbf{a}_{k+n}, \\
d \mathbf{a}_{i+n} & =\sum_{k=1}^{n} \phi_{i k} \mathbf{a}_{k}-\sum_{k=1}^{n} \omega_{k i} \mathbf{a}_{k+n}
\end{aligned}
$$

where as a consequence of the normalization conditions (2.6), the one-forms $\theta_{i j}$ and $\phi_{i j}$ satisfy

$$
\theta_{i j}=\theta_{j i}, \quad \phi_{i j}=\phi_{j i}, \quad \text { for all } \quad 1 \leq i, j \leq n .
$$

Thus, the matrix valued 1-form

$$
\Theta=\left(\begin{array}{cc}
\omega & \theta \\
\phi & -\omega^{t}
\end{array}\right),
$$

takes values in the Lie algebra $\mathfrak{s p}(2 n, \mathbb{R})$. 


\section{Curves.}

The main object of study of this paper is the differential geometry of curves $C \subset \mathbf{R}^{2 n}$ under the group of rigid symplectic motions. We consider regularly parametrized smooth curves $\mathbf{z}: I \rightarrow \mathbb{R}^{2 n}$ defined on an open interval $I \subset \mathbf{R}$ whose second-order osculating spaces satisfy the non-degeneracy condition

$$
\langle\dot{\mathbf{z}} ; \ddot{\mathbf{z}}\rangle \neq 0, \quad \text { for all } \quad t \in I \text {. }
$$

We shall refer to such curves as symplectic regular curves. With no loss of generality, we may assume that the left-hand-side in (3.1) is positive.

Definition 1 Let $t_{0} \in I$. The symplectic arc length $s$ of a symplectic regular curve $\mathbf{z}$ starting at $t_{0}$ is defined by $y^{2}$ To keep

$$
s(t)=\int_{t_{0}}^{t}\langle\dot{\mathbf{z}} ; \ddot{\mathbf{z}}\rangle^{1 / 3} d t
$$

for $t \in I$. We say that a symplectic regular curve $\mathbf{z}$ is parametrized by symplectic arc length if

$$
\int_{t_{1}}^{t_{2}}\langle\dot{\mathbf{z}} ; \ddot{\mathbf{z}}\rangle^{1 / 3} d t=t_{2}-t_{1} \quad \text { for all } \quad t_{1}, t_{2} \in I, \quad t_{1} \leq t_{2}
$$

It is easily seen that the condition (3.3) is equivalent to

$$
\langle\dot{\mathbf{z}} ; \ddot{\mathbf{z}}\rangle=1, \quad \text { for all } \quad t \in I \text {. }
$$

It is noteworthy that the symplectic arc length parameter coincides with the equiaffine arclength for planar curves. The latter is geometrically interpreted in

\footnotetext{
${ }^{2}$ As defined, the symplectic arc length could be negative. This can be easily overcome by replacing $\langle\dot{\mathbf{z}} ; \ddot{\mathbf{z}}\rangle$ by its absolute value, but we will not to do this in order to keep our subsequent computations as simple as possible.
} 
terms of the area of the support triangle attached to each point of the curve [4].

Remark: We use dots to denote derivatives with respect to an arbitrary parametrization $t$, reserving primes to indicate derivatives with respect to symplectic arc length, which from here onwards we denote by $s$. Although $s$ depends on the starting point $t_{0}$, the symplectic arc length element

$$
d s=\langle\dot{\mathbf{z}} ; \ddot{\mathbf{z}}\rangle^{1 / 3} d t
$$

and associated arc length derivative operator

$$
\frac{d}{d s}=\langle\dot{\mathbf{z}} ; \ddot{\mathbf{z}}\rangle^{-1 / 3} \frac{d}{d t}
$$

do not.

The following proposition shows that there is no loss of generality in assuming that any symplectic regular curve can be re-parametrized by symplectic arclength.

Proposition 1 Let $\mathbf{z}: I \rightarrow \mathbb{R}^{2 n}$ be a symplectic regular curve and let $s$ be the corresponding symplectic arc length function. Then there exists an inverse function $h$ of $s$ defined on $\widetilde{I}=s(I)$ and a reparametrization $\mathbf{w}=\mathbf{z} \circ h: \widetilde{I} \rightarrow \mathbb{R}^{2 n}$ of $\mathbf{z}$ which is parametrized by symplectic arclength.

Proof: Since $\mathbf{z}$ is symplectic regular, it follows that $s$ is a strictly monotone function of $t$ in the interval $I$, so that there exists an inverse function $h: \widetilde{I} \rightarrow I$ of $s$. Furthermore, we have

$$
\mathbf{w}^{\prime}=\frac{d \mathbf{w}}{d s}=\langle\dot{\mathbf{z}} ; \ddot{\mathbf{z}}\rangle^{-1 / 3} \dot{\mathbf{z}}, \quad \mathbf{w}^{\prime \prime}=\frac{d^{2} \mathbf{w}}{d s^{2}}=\langle\dot{\mathbf{z}} ; \ddot{\mathbf{z}}\rangle^{-2 / 3} \ddot{\mathbf{z}}+\frac{d^{2} h}{d s^{2}} \dot{\mathbf{z}},
$$

so that

$$
\left\langle\frac{d \mathbf{w}}{d s} ; \frac{d^{2} \mathbf{w}}{d s^{2}}\right\rangle=\left\langle\frac{\dot{\mathbf{z}}}{\langle\dot{\mathbf{z}} ; \ddot{\mathbf{z}}\rangle^{1 / 3}} ; \frac{\ddot{\mathbf{z}}}{\langle\dot{\mathbf{z}} ; \ddot{\mathbf{z}}\rangle^{2 / 3}}\right\rangle=1
$$




\section{$4 \quad$ Adapted symplectic frames}

\section{- Frenet formulas.}

Let $\mathbf{z}: I \rightarrow \mathbb{R}^{2 n}$ be a symplectic regular curve parametrized by symplectic arc length. To any such curve, we associate an adapted symplectic frame $\left(\mathbf{a}_{1}, \ldots, \mathbf{a}_{2 n}\right)$. This frame is defined recursively. We let

$$
\mathbf{a}_{1}=\frac{d \mathbf{z}}{d s}, \quad \mathbf{a}_{n+1}=\frac{d^{2} \mathbf{z}}{d s^{2}}
$$

and define

$$
K_{1}=\left\langle\frac{d \mathbf{a}_{n+1}}{d s} ; \mathbf{a}_{n+1}\right\rangle .
$$

Then, for each $1 \leq j \leq n-2$, we let

$$
\mathbf{a}_{j+1}=\frac{d \mathbf{a}_{n+j}}{d s}-K_{j} \mathbf{a}_{j}-\left(1-\delta_{j 1}\right) \mathbf{a}_{j-1}, \quad H_{j+1}=-\left\langle\frac{d \mathbf{a}_{j+1}}{d s} ; \mathbf{a}_{j+1}\right\rangle .
$$

If $H_{j+1} \neq 0$ for all $1 \leq j \leq n-2$, we let

$$
\mathbf{a}_{n+j+1}=\frac{1}{H_{j+1}} \frac{d \mathbf{a}_{j+1}}{d s}, \quad K_{j+1}=\left\langle\frac{d \mathbf{a}_{n+j+1}}{d s} ; \mathbf{a}_{n+j+1}\right\rangle .
$$

Finally, we define the frame vectors $\mathbf{a}_{n}$ and $\mathbf{a}_{2 n}$ and the structure functions $H_{n}$ and $K_{n}$ as follows. We let

$$
\mathbf{a}_{n}=\frac{d \mathbf{a}_{2 n-1}}{d s}-K_{n-1} \mathbf{a}_{n-1}-\left(1-\delta_{n-1,1}\right) \mathbf{a}_{n-2},
$$

and define $\mathbf{a}_{2 n}$ uniquely by the orthonormality relations

$$
\begin{array}{ll}
\left\langle\mathbf{a}_{i} ; \mathbf{a}_{2 n}\right\rangle=0, \quad & \left\langle\mathbf{a}_{n+i} ; \mathbf{a}_{2 n}\right\rangle=0, \\
\left\langle\mathbf{a}_{n} ; \mathbf{a}_{2 n}\right\rangle=1, & \left\langle\frac{d \mathbf{a}_{n}}{d s} ; \mathbf{a}_{2 n}\right\rangle=0 .
\end{array}
$$


Furthermore, we let

$$
H_{n}=-\left\langle\frac{d \mathbf{a}_{n}}{d s} ; \mathbf{a}_{n}\right\rangle, \quad K_{n}=\left\langle\frac{d \mathbf{a}_{2 n}}{d s} ; \mathbf{a}_{2 n}\right\rangle .
$$

We now check that the frame we have just defined is indeed a symplectic frame along the image of $\mathbf{z}$, in other words that the orthonormality conditions (2.6) are satisfied at every point of the image of $\mathbf{z}$.

Proposition 2 Let $\mathbf{z}: I \rightarrow \mathbb{R}^{2 n}$ be a symplectic regular curve which is parametrized by symplectic arc length and such that $H_{j+1} \neq 0$ for all $1 \leq j \leq n-2$. Then the frame $\left(\mathbf{a}_{1}, \ldots, \mathbf{a}_{2 n}\right)$ defined along the image of $\mathbf{z}$ is symplectic.

Proof: The proof proceeds by induction. By definition, we have

$$
\left\langle\mathbf{a}_{1} ; \mathbf{a}_{1}\right\rangle=\left\langle\mathbf{a}_{n+1} ; \mathbf{a}_{n+1}\right\rangle=0, \quad\left\langle\mathbf{a}_{1} ; \mathbf{a}_{n+1}\right\rangle=1
$$

Assume that for some $1 \leq j \leq n-2$ and for all $1 \leq i, l \leq j$, the frame vectors $\mathbf{a}_{1}, \ldots, \mathbf{a}_{j}, \ldots, \mathbf{a}_{n+1}, \ldots, \mathbf{a}_{n+j}$ defined above satisfy the orthonormality relations

$$
\left\langle\mathbf{a}_{i} ; \mathbf{a}_{l}\right\rangle=0, \quad\left\langle\mathbf{a}_{i} ; \mathbf{a}_{n+l}\right\rangle=\delta_{i l}=-\left\langle\mathbf{a}_{n+l} ; \mathbf{a}_{i}\right\rangle, \quad\left\langle\mathbf{a}_{n+i} ; \mathbf{a}_{n+l}\right\rangle=0 .
$$

We have to show that the frame vectors $\mathbf{a}_{j+1}$ and $\mathbf{a}_{n+j+1}$ satisfy the required orthonormality conditions relative to the frame vectors $\mathbf{a}_{1}, \ldots, \mathbf{a}_{j}, \ldots, \mathbf{a}_{n+1}$, $\ldots, \mathbf{a}_{n+j}$. We will verify a few of the relations and leave the remaining ones to the reader as an exercise. We have, using (4.3), (4.4), and our induction hypothesis, that

$$
\begin{aligned}
\left\langle\mathbf{a}_{j+1} ; \mathbf{a}_{l}\right\rangle & =\left\langle\frac{d \mathbf{a}_{n+j}}{d s}-K_{j} \mathbf{a}_{j}-\left(1-\delta_{j 1}\right) \mathbf{a}_{j-1} ; \mathbf{a}_{l}\right\rangle \\
& =\left\langle\frac{d \mathbf{a}_{n+j}}{d s} ; \mathbf{a}_{l}\right\rangle=-\left\langle\mathbf{a}_{n+j} ; \frac{d \mathbf{a}_{l}}{d s}\right\rangle=H_{l}\left\langle\mathbf{a}_{n+j} ; \mathbf{a}_{n+l}\right\rangle=0,
\end{aligned}
$$


as required. Similarly, we have

$$
\left\langle\mathbf{a}_{j+1} ; \mathbf{a}_{n+l}\right\rangle=-\left\langle\mathbf{a}_{n+j} ; \frac{d \mathbf{a}_{n+l}}{d s}\right\rangle-K_{j} \delta_{j l}-\left(1-\delta_{j i}\right) \delta_{j-1 l}
$$

If $l=j$, then we obtain, using (4.4), that the left-hand side of (4.9) vanishes, as required. If $2 \leq l \leq j$, then (4.3) and (4.9) together with the induction hypothesis imply that

$$
\left\langle\mathbf{a}_{j+1} ; \mathbf{a}_{n+l}\right\rangle=\delta_{j, l+1}-\delta_{j, l-1}+\delta_{l 1} \delta_{j, l-1}-\delta_{j-1, l}+\delta_{j 1} \delta_{j-1, l}=0
$$

Finally, if $l=1<j$, we have, using (4.3),

$$
\begin{aligned}
\left\langle\mathbf{a}_{j+1} ; \mathbf{a}_{n+l}\right\rangle & =-\left\langle\mathbf{a}_{n+j} ; \frac{d \mathbf{a}_{n+1}}{d s}\right\rangle-K_{j} \delta_{j 1}-\left(1-\delta_{j 1}\right) \delta_{j-1,1} \\
& =-\left\langle\mathbf{a}_{n+j} ; \mathbf{a}_{2}+K_{1} \mathbf{a}_{1}\right\rangle-\delta_{j-1,1}=0 .
\end{aligned}
$$

The proof of the remaining orthonormality conditions is similar, and will therefore be omitted.

The adapted symplectic frame that we have just defined for any symplectic regular curve can be thought of as a Frenet frame. The structure equations for this frame, which we now derive, show that the $2 n-1$ functions $H_{2}, \ldots, H_{n}$ and $K_{1}, \ldots, K_{n}$ play the role of symplectic curvatures for symplectic regular curves. We remark that it follows from the definitions (4.2), (4.3), (4.4) and (4.7) of the symplectic curvatures $H_{2}, \ldots, H_{n}$ and $K_{1}, \ldots, K_{n}$ can be interpreted geometrically as giving a measure of the deviation of certain 2-planes spanned by pairs of vectors in the symplectic Frenet frame from being Lagrangian. For example, if $K_{1}=0$, then the 2-plane spanned by $\frac{d \mathbf{a}_{n+1}}{d s}$ and $\mathbf{a}_{n+1}$ will be Lagrangian.

Proposition 3 Let $\mathbf{z}: I \rightarrow \mathbb{R}^{2 n}$ be a symplectic regular curve which is parametrized by symplectic arc length and such that ${ }^{3} H_{j+1} \neq 0$ for all $1 \leq j \leq n-2$.

\footnotetext{
${ }^{3}$ Here, and below, when $n=2$, this nondegeneracy condition is empty.
} 
Then the frame $\left(\mathbf{a}_{1}, \ldots, \mathbf{a}_{2 n}\right)$ defined along the image of $\mathbf{z}$ satisfies the structure equations

$$
\begin{aligned}
& \frac{d \mathbf{a}_{1}}{d s}=\mathbf{a}_{n+1}, \quad \frac{d \mathbf{a}_{i}}{d s}=H_{i} \mathbf{a}_{n+i}, \quad 2 \leq i \leq n, \\
& \frac{d \mathbf{a}_{n+1}}{d s}=K_{1} \mathbf{a}_{1}+\mathbf{a}_{2}, \quad \frac{d \mathbf{a}_{n+j}}{d s}=\mathbf{a}_{j-1}+K_{j} \mathbf{a}_{j}+\mathbf{a}_{j+1}, \quad 2 \leq j \leq n-1, \\
& \frac{d \mathbf{a}_{2 n}}{d s}=\mathbf{a}_{n-1}+K_{n} \mathbf{a}_{n} \text {. }
\end{aligned}
$$

Proof: Again, we will only establish a few of the above structure equations, because their proofs are all quite similar. The first of the structure equations is a direct consequence of the definition (4.1). Likewise, the second set of structure equations are, for $2 \leq j \leq n-1$, direct consequences of the definition (4.4). To prove the structure equation

$$
\frac{d \mathbf{a}_{n}}{d s}=H_{n} \mathbf{a}_{2 n}
$$

we first let

$$
\frac{d \mathbf{a}_{n}}{d s}=\sum_{i=1}^{n} \alpha_{i} \mathbf{a}_{i}+\sum_{i=1}^{n} \beta_{i} \mathbf{a}_{n+i} .
$$

In view of (4.6), we immediately deduce that $\alpha_{n}=0$. For $1 \leq i \leq n-1$, we obtain, using (4.3) and (4.5),

$$
\begin{aligned}
\alpha_{i}=\left\langle\frac{d \mathbf{a}_{n}}{d s} ; \mathbf{a}_{n+i}\right\rangle & =-\left\langle\mathbf{a}_{n} ; \frac{d \mathbf{a}_{n+i}}{d s}\right\rangle \\
& =-\left\langle\mathbf{e}_{n} ; \mathbf{a}_{i+1}+K_{i} \mathbf{a}_{i}+\left(1-\delta_{i 1} \mathbf{a}_{i-1}\right)\right\rangle=0 .
\end{aligned}
$$

Then (4.7) implies that $\beta_{n}=H_{n}$. Finally, for $1 \leq i \leq n-1$, we have, using (4.4) and (4.1),

$$
\beta_{i}=-\left\langle\frac{d \mathbf{a}_{n}}{d s} ; \mathbf{a}_{i}\right\rangle=\left\langle\mathbf{a}_{n} ; \frac{d \mathbf{a}_{i}}{d s}\right\rangle=0
$$


The proofs of the remaining structure equations are similar.

The system of equations (4.9) can be written in matrix notation as

$$
\left(\begin{array}{c}
\frac{d \mathbf{a}_{1}}{d s} \\
\vdots \\
\frac{d \mathbf{a}_{2 n}}{d s}
\end{array}\right)=B\left(\begin{array}{c}
\mathbf{a}_{1} \\
\vdots \\
\mathbf{a}_{2 n}
\end{array}\right)
$$

where

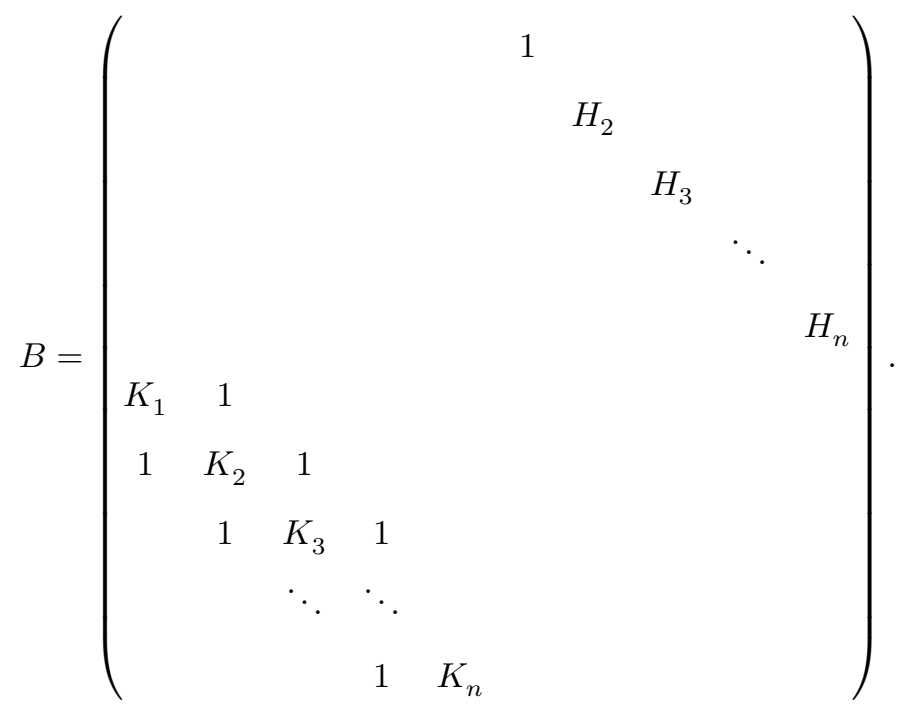

Using the Frenet formulas, we are able to prove an existence and uniqueness theorem for symplectic regular curves which is similar to the standard theorem for curves in Euclidean geometry.

Theorem 1 Let $H_{2}, \ldots, H_{n}$ and $K_{1}, \ldots, K_{n}$ be $2 n-1$ smooth real valued functions defined on an interval $I$ with $H_{j} \neq 0$ for all $2 \leq j \leq n-1$. There exists, up to a rigid symplectic motion of $\left(\mathbb{R}^{2 n}, \Omega\right)$, a unique symplectic regular curve $\mathbf{z}: I \rightarrow \mathbb{R}^{2 n}$, parametrized by symplectic arc length, whose local symplectic invariants are the given functions $H_{2}, \ldots, H_{n}$ and $K_{1}, \ldots, K_{n}$. 
Proof: The method of proof is identical to the one used for curves in Euclidean geometry. The existence of a Frenet frame satisfying the structure equations (4.10) follows from the global existence theorem for linear systems of first-order ordinary differential equations with smooth coefficients, with initial conditions satisfying (2.6). The uniqueness is obtained by deriving a system of first-order ordinary differential equations for the symplectic inner products $\left\langle\mathbf{a}_{a} ; \mathbf{a}_{b}\right\rangle$ for $1 \leq a, b, \leq 2 n$, and showing that any solution of (4.10) will always gives rise to a solution of this system. The system is given by

$$
\begin{gathered}
\frac{d}{d s}\left\langle\mathbf{a}_{i} ; \mathbf{a}_{j}\right\rangle=\delta_{i 1}\left\langle\mathbf{a}_{n+1} ; \mathbf{a}_{j}\right\rangle+\left(1-\delta_{i 1}\right) H_{i}\left\langle\mathbf{a}_{n+i} ; \mathbf{a}_{j}\right\rangle \\
+\delta_{j 1}\left\langle\mathbf{a}_{i} ; \mathbf{a}_{n+1}\right\rangle+\left(1-\delta_{j 1}\right) H_{j}\left\langle\mathbf{a}_{i} ; \mathbf{a}_{n+j}\right\rangle, \\
\frac{d}{d s}\left\langle\mathbf{a}_{i} ; \mathbf{a}_{n+j}\right\rangle=\delta_{i 1}\left\langle\mathbf{a}_{n+1} ; \mathbf{a}_{n+j}\right\rangle+\left(1-\delta_{i 1}\right) H_{i}\left\langle\mathbf{a}_{n+i} ; \mathbf{a}_{n+j}\right\rangle \\
+\left(1-\delta_{j 1}\right)\left\langle\mathbf{a}_{i} ; \mathbf{a}_{j-1}\right\rangle+K_{j}\left\langle\mathbf{a}_{i} ; \mathbf{a}_{j}\right\rangle+\left(1-\delta_{j n}\right)\left\langle\mathbf{a}_{i} ; \mathbf{a}_{j+1}\right\rangle, \\
\frac{d}{d s}\left\langle\mathbf{a}_{n+i} ; \mathbf{a}_{j}\right\rangle=\left(1-\delta_{i 1}\right)\left\langle\mathbf{a}_{i-1} ; \mathbf{a}_{j}\right\rangle+K_{i}\left\langle\mathbf{a}_{i} ; \mathbf{a}_{j}\right\rangle+\left(1-\delta_{i n}\right)\left\langle\mathbf{a}_{i+1} ; \mathbf{a}_{j}\right\rangle \\
+\delta_{1 j}\left\langle\mathbf{a}_{n+i} ; \mathbf{a}_{n+1}\right\rangle+\left(1-\delta_{i j}\right) H_{j}\left\langle\mathbf{a}_{n+i} ; \mathbf{a}_{n+j}\right\rangle \\
\frac{d}{d s}\left\langle\mathbf{a}_{n+i} ; \mathbf{a}_{n+j}\right\rangle=\left(1-\delta_{i 1}\right)\left\langle\mathbf{a}_{i-1} ; \mathbf{a}_{n+j}\right\rangle+K_{i}\left\langle\mathbf{a}_{i} ; \mathbf{a}_{n+j}\right\rangle \\
+\left(1-\delta_{i n}\right)\left\langle\mathbf{a}_{i+1} ; \mathbf{a}_{n+j}\right\rangle+\left(1-\delta_{j 1}\right)\left\langle\mathbf{a}_{n+i} ; \mathbf{a}_{j-1}\right\rangle \\
+K_{j}\left\langle\mathbf{a}_{n+i} ; \mathbf{a}_{j}\right\rangle+\left(1-\delta_{j n}\right)\left\langle\mathbf{a}_{n+i} ; \mathbf{a}_{j+1}\right\rangle .
\end{gathered}
$$

It is now straightforward to show that any frame satisfying the orthonormality conditions (2.6) will solve this system. Hence the solution of (4.10) with the given initial condition satisfies the orthonormality condition.

We conclude this section by giving the parametric expressions of a couple of the invariants that we have just defined. These expressions are fairly simple when the symplectic regular curve is parametrized by symplectic arc length, but they become quite complicated in a more general parametrization. For example, $\mathbf{z}(s)$ denotes a symplectic regular curve parametrized by arc length, meaning 
that $\left\langle\mathbf{z}^{\prime} ; \mathbf{z}^{\prime \prime}\right\rangle=1$ then we have

$$
K_{1}=\left\langle\frac{d^{3} \mathbf{z}}{d s^{3}} ; \frac{d^{2} \mathbf{z}}{d s^{2}}\right\rangle, \quad \text { and } \quad H_{2}=-\left\langle\frac{d^{4} \mathbf{z}}{d s^{4}} ; \frac{d^{3} \mathbf{z}}{d s^{3}}\right\rangle-K_{1}^{2} .
$$

We note that for a symplectic regular curve parametrized by symplectic arc length, $H_{j}$ will be of order $2 j$ and $K_{j}$ of order $2 j+1$.

\section{The Method of Moving Coframes.}

In this section, we review the method of moving coframes developed in [9] in a general setting. The following section will apply the method to the context at hand: symplectic curves. Throughout this section, $G$ will denote an $r$-dimensional Lie group that acts smoothly on an $m$-dimensional manifold $M$. We also assume, for the purposes of exposition, that $G$ acts transitively on $M$, although this is inessential for the applicability of the moving frame method.

We consider the lifted action $(z, h) \mapsto(g \cdot z, g \cdot h)$ defined on the trivial principal bundle $\mathcal{B}=M \times G$. A complete system of functionally independent invariants on $\mathcal{B}$ is provided by the components of $w(z, g)=g^{-1} \cdot z$, which are defined as lifted invariants. A complete system of invariant differential forms is provided by the differentials $d w$ of the lifted invariants along with the (pulledback) left-invariant Maurer-Cartan forms $\boldsymbol{\mu}$ on $G$.

We are interested in computing the differential invariants of $p$-dimensional submanifolds $\varphi: T \rightarrow M$ where $T \subset \mathbf{R}^{p}$ is the parameter space. (Since we are dealing with local properties, there is no loss in generality in assuming that $T$ is a subset of Euclidean space.) Two submanifolds are said to be (locally) equivalent if there exists a group transformation $g \in G$ and a local diffeomorphism $\tau: T \rightarrow$ $T$ such that $\widetilde{\varphi}=g \cdot(\varphi \circ \tau)$. This is the same as requiring equivalence under the pseudo-group $\mathcal{G}=G \times \mathcal{D}(T)$, where $\mathcal{D}(T)$, the reparametrization pseudo-group, 
denotes the full diffeomorphism of the parameter space. Again, the moving coframe method can equally well handle restricted reparametrization groups and pseudo-groups, but we will focus our attention to the simplest case here.

In order to encode the reparametrizations using differential forms, we introduce the extended principal bundle $\widetilde{\mathcal{B}}=(T \times M) \times(\mathrm{GL}(p) \times G)$. Let $\mathcal{I}=\{w, d w, \boldsymbol{\mu}, \boldsymbol{\omega}\}$ denote the extended coframe consisting of the lifted invariants $w=g^{-1} \cdot z$, their derivatives, the (pulled-back) left-invariant MaurerCartan forms $\boldsymbol{\mu}$, and the parameter one-forms

$$
\boldsymbol{\omega}=\Lambda \cdot d \mathbf{t}=\sum_{i, j} \lambda_{j}^{i} d t^{j}
$$

where $\Lambda \in \operatorname{GL}(p)$ and $t=\left(t^{1}, \ldots, t^{p}\right)$ are local coordinates on $T$. These oneforms play the role of Maurer-Cartan forms for the reparametrization pseudogroup, [9]. It is not hard to see that $\mathcal{I}$ forms a $\mathcal{G}$-coframe, in the terminology of [9], which means that a diffeomorphism $\Psi: \widetilde{\mathcal{B}} \rightarrow \widetilde{\mathcal{B}}$ is a symmetry of $\mathcal{I}$, so $\Psi^{*} \mathcal{I}=\mathcal{I}$, if and only if $\Psi$ is a lift of an element $\psi \in \mathcal{G}$.

We prolong the action of $\mathcal{G}$ to the infinite jet bundle $\mathrm{J}^{\infty}=\mathrm{J}^{\infty}(T, M)$ of maps $\varphi: T \rightarrow M$. There is a natural splitting of the space of one-forms on $\mathrm{J}^{\infty}$ into horizontal and vertical or contact components, and we let $d=d_{H}+d_{V}$ be the induced splitting of the differential, which induces the variational bicomplex structure over $\mathrm{J}^{\infty}$, cf. [2]. There is a corresponding prolonged principal bundle $\widetilde{\mathcal{B}}^{(n)}=$ $\mathrm{J}^{n} \times(\mathrm{GL}(p) \times G)$ and prolonged differential system $\mathcal{I}^{(n)}=w^{(n)}, d w^{(n)}, \boldsymbol{\mu}, \boldsymbol{\omega}$ which now includes the lifted differential invariants $w^{(n)}=\left(g^{(n)}\right)^{-1} \cdot z^{(n)}$ and their differentials. Here $z^{(n)}=\left(\ldots z_{J}^{\alpha} \ldots\right)$ are the usual jet bundle coordinates induced by coordinates $t=\left(t^{1}, \ldots, t^{p}\right)$ on $T$ and $z=\left(z^{1}, \ldots, z^{m}\right)$ on $M$, while $g^{(n)}$ denotes the prolonged action of $G$. We note that, under the bigrading of the space of one-forms, $d w^{(n)}=w^{(n+1)} d \mathbf{t}+d_{V} w^{(n)}$, so that the horizontal components of $d w^{(n)}$ provide the order $n+1$ lifted differential invariants. 
The goal of the method of moving coframes, $[8,9]$, is to construct a $\mathcal{G}-$ equivariant section $\sigma^{(n)}: \mathrm{J}^{n} \rightarrow \widetilde{\mathcal{B}}^{(n)}$. Once $\sigma^{(n)}$ is specified, it will pull-back $\mathcal{G}$-invariant quantities on $\widetilde{\mathcal{B}}^{(n)}$ to $\mathcal{G}$-invariant quantities on $\mathrm{J}^{n}$. In particular, $\left(\sigma^{(n)}\right)^{*} w^{(n)}=I^{(n)}$ form a complete system of $n^{\text {th }}$ order differential invariants, while $\left(\sigma^{(n)}\right)^{*} \boldsymbol{\omega}=\boldsymbol{\omega}$ form a complete system of invariant horizontal one-forms — the "Frenet coframe" for our submanifolds. Higher order differential invariants can, alternatively, be found by invariant differentiation with respect to the invariant horizontal one-forms $\boldsymbol{\omega}$.

In the moving coframe procedure, one begins by normalizing the order zero invariants by setting them equal to suitably chosen constants:

$$
w=g^{-1} \cdot z=c
$$

(If $G$ does not act transitively on $M$, then we can only normalize some of the order zero invariants, and the remainder will lead to ordinary invariants.) We solve the $m \leq r$ order 0 normalization equations (5.1) for $m$ of the group parameters. Substituting these formulae into the Maurer-Cartan forms $\boldsymbol{\mu}, \boldsymbol{\omega}$ will lead to certain linear dependencies among them

$$
\mu^{\kappa}=\sum_{\alpha} J_{\alpha}^{\kappa} \mu^{\alpha}+\sum_{i} K_{i}^{\kappa} \omega^{i}
$$

The coefficients $J_{\alpha}^{\kappa}, K_{i}^{\kappa}$ of these linear dependencies are lifted invariants, and so can be normalized to suitable constants. One then iterates the process until there are no remaining group parameters. The nonconstant coefficients of the resulting linear dependencies among the pulled-back Maurer-Cartan forms will give a complete system of differential invariants. 


\section{Symplectic Curves.}

As above, the affine symplectic group $G=\operatorname{Sp}(2 n, \mathbb{R}) \ltimes \mathbf{R}^{2 n}$ acts on $M=\mathbf{R}^{2 n}$ via rigid symplectic motions (2.5). We will often write the symplectic matrix $A=$ $\left(\mathbf{a}_{1}, \ldots, \mathbf{a}_{2 n}\right)$ in column form. We note that the columns satisfy the symplecticity conditions

$$
\left\langle\mathbf{a}_{i} ; \mathbf{a}_{j}\right\rangle= \begin{cases}1, & j=i+n \\ -1, & i=j+n \\ 0, & \text { otherwise }\end{cases}
$$

where $\langle\cdot ; \cdot\rangle$ is the standard symplectic inner product $(2.2)$ on $\mathbf{R}^{2 n}$. We study the induced action on suitably regular curves $\mathbf{z}: I \rightarrow M$. Two such curves are equivalent if the first can be mapped to the second under a rigid symplectic motion up to a reparametrization.

Consider the action of the Cartesian product pseudo-group $\mathcal{G}=G \times \mathcal{D}(1)$ on the extended principal bundle $\widetilde{\mathcal{B}}=\mathbf{R}^{2 n} \times(G \times \mathrm{GL}(1))$. The left-invariant Maurer-Cartan forms are

$$
\boldsymbol{\mu}=\left(\boldsymbol{\mu}_{1}, \ldots, \boldsymbol{\mu}_{2 n}\right)=A^{-1} d A, \quad \boldsymbol{\nu}=A^{-1} d \mathbf{b}, \quad \omega=\lambda d t
$$

where $\boldsymbol{\mu}_{k}$ denotes the $k^{\text {th }}$ column of the Maurer-Cartan form matrix $\boldsymbol{\mu}$. The final "Maurer-Cartan form" $\omega$ arises from the reparametrization pseudo-group $t \mapsto \tau(t)$, and $\lambda \neq 0$ is a free parameter.

We will show how to use the moving coframe method outlined in Section 5 in this particular context. The initial step in the algorithm is to normalize the order zero lifted invariants (5.1), which, for the affine symplectic group action, are

$$
A^{-1}(\mathbf{z}-\mathbf{b})=\mathbf{0} \quad \text { so that } \quad \mathbf{b}=\mathbf{z} .
$$

Therefore, as usual, the translational component of the (left) moving frame can 
be identified as the point on the curve.

Next, we substitute the initial normalizations (6.3) into the Maurer-Cartan forms (6.2). Any resulting linear dependencies will give additional lifted differential invariants. Substituting $\mathbf{b}=\mathbf{z}$ into the translation Maurer-Cartan forms gives

$$
\boldsymbol{\nu}=A^{-1} d \mathbf{z}=A^{-1} \dot{\mathbf{z}} d t=\frac{A^{-1} \dot{\mathbf{z}}}{\lambda} \omega
$$

The coefficients $\lambda^{-1} A^{-1} \dot{\mathbf{z}}$ in (6.4) are lifted invariants, and can be normalized to any convenient constant values. We choose to set the first component equal to 1 and the rest equal to 0 . (They can't all be set equal to zero unless $\dot{\mathbf{z}}=\mathbf{0}$, which we exclude - along with certain other "nondegeneracy" conditions that will show up along the way.) In other words, we solve

$$
\frac{A^{-1} \dot{\mathbf{z}}}{\lambda}=\mathbf{e}_{1}
$$

where $\mathbf{e}_{1}, \ldots, \mathbf{e}_{2 n}$ are used to denote the standard basis vectors of $\mathbf{R}^{2 n}$. Multiplying by $A$, we conclude that its first column $\mathbf{a}_{1}=A \mathbf{e}_{1}$ has been normalized to

$$
\mathbf{a}_{1}=\frac{\dot{\mathbf{z}}}{\lambda}, \quad \text { and so } \quad \boldsymbol{\nu}=\omega \mathbf{e}_{1}
$$

We next substitute the normalized value (6.5) for the first column of $A$ into the remaining Maurer-Cartan forms. In particular, the first column of the Maurer-Cartan form matrix is

$$
\boldsymbol{\mu}_{1}=A^{-1} d \mathbf{a}_{1}=\frac{A^{-1} \ddot{\mathbf{z}}}{\lambda^{2}} \omega-\frac{A^{-1} \dot{\mathbf{z}}}{\lambda^{2}} d \lambda=\frac{A^{-1} \ddot{\mathbf{z}}}{\lambda^{2}} \omega-\frac{d \lambda}{\lambda} \mathbf{e}_{1}
$$

Now since $\omega=\lambda d t$ is $\mathcal{G}$-invariant, so is

$$
d \omega=\frac{d \lambda}{\lambda} \wedge \omega
$$


This implies the $\mathcal{G}$-invariance of the one-form

$$
\eta=\frac{d \lambda}{\lambda}+\alpha \omega
$$

in which $\alpha$ denotes an additional pseudo-group parameter, which represents the second order jet of the reparametrization pseudo-group. Substituting (6.7) back into (6.6), we find

$$
\boldsymbol{\mu}_{1}=\left(\frac{A^{-1} \ddot{\mathbf{z}}}{\lambda^{2}}+\alpha \mathbf{e}_{1}\right) \omega-\eta \mathbf{e}_{1}
$$

The coefficients of $\omega$ are invariant, and will be normalized to

$$
\frac{A^{-1} \ddot{\mathbf{z}}}{\lambda^{2}}+\alpha \mathbf{e}_{1}=\mathbf{e}_{n+1} .
$$

Thus,

$$
\mathbf{a}_{n+1}=\frac{\ddot{\mathbf{z}}}{\lambda^{2}}+\alpha \mathbf{a}_{1} .
$$

But the symplecticity requirement (6.1) implies that

$$
1=\left\langle\mathbf{a}_{1} ; \mathbf{a}_{n+1}\right\rangle=\frac{\langle\dot{\mathbf{z}} ; \ddot{\mathbf{z}}\rangle}{\lambda^{3}},
$$

and therefore we are led to normalize

$$
\lambda=\sqrt[3]{\langle\dot{\mathbf{z}} ; \ddot{\mathbf{z}}\rangle} .
$$

In particular, we must impose the nondegneracy requirement

$$
\langle\dot{\mathbf{z}} ; \ddot{\mathbf{z}}\rangle \neq 0
$$

for our choice of normalization to be applicable.

Remark: Most curves that fail to satisfy the nondegeneracy requirement (6.11) 
can still be treated by the moving frame method, but require use of a higher order moving frame, [9]. The only curves that do not admit any moving frame whatsoever are the totally singular curves, meaning those for which the group $G$ fails to act locally freely on their jets of any order. Moreover, according to the results of [11], a curve $C$ is totally singular if and only if its symmetry group $G_{C}=\{g \in G \mid g \cdot C=C\}$ has dimension $\operatorname{dim} G \geq 2$. If $\operatorname{dim} G_{C}=1$, then the curve has all constant differential invariants and is contained in the orbit of a suitable one-parameter subgroup, while if $\operatorname{dim} G_{C}=0$ then the curve has at least one non-constant differential invariant and only a discrete symmetry group. See Section 7 for further results in this direction.

The resulting invariant one-form

$$
\omega \longmapsto d s=\sqrt[3]{\langle\dot{\mathbf{z}} ; \ddot{\mathbf{z}}\rangle} d t
$$

recovers the symplectic arc-length element (3.5). Note that we can now write

$$
\mathbf{a}_{1}=\mathbf{z}^{\prime} \equiv \mathbf{z}_{1}, \quad \mathbf{a}_{n+1}=\mathbf{z}^{\prime \prime}+\left(\alpha+\frac{\lambda^{\prime}}{\lambda}\right) \mathbf{z}^{\prime}=\mathbf{z}^{\prime \prime}+\left(\alpha+(\log \lambda)^{\prime}\right) \mathbf{z}^{\prime}
$$

where, as above, we use primes to denote arc length derivatives, with

$$
\mathbf{z}^{\prime}=\frac{\dot{\mathbf{z}}}{\lambda}, \quad \mathbf{z}^{\prime \prime}=\frac{\ddot{\mathbf{z}}}{\lambda^{3}}-\frac{\dot{\lambda} \dot{\mathbf{z}}}{\lambda^{2}}, \quad \lambda^{\prime}=\frac{\dot{\lambda}}{\lambda}=\frac{\langle\dot{\mathbf{z}} ; \ddot{\mathbf{z}}\rangle}{3\langle\dot{\mathbf{z}} ; \ddot{\mathbf{z}}\rangle}
$$

and so on. Our normalization (6.9) requires

$$
\left\langle\mathbf{a}_{1} ; \mathbf{a}_{n+1}\right\rangle=\left\langle\mathbf{z}^{\prime} ; \mathbf{z}^{\prime \prime}\right\rangle=1
$$


Continuing the procedure, we next consider the Maurer-Cartan forms

$$
\begin{aligned}
\boldsymbol{\mu}_{n+1} & =A^{-1} d \mathbf{a}_{n+1} \\
& =A^{-1}\left[\mathbf{z}^{\prime \prime \prime}+\left(\alpha+(\log \lambda)^{\prime}\right) \mathbf{z}^{\prime \prime}+(\log \lambda)^{\prime \prime} \mathbf{z}^{\prime}\right] \omega+\mathbf{e}_{1} d \alpha
\end{aligned}
$$

where $\lambda$ is given by (6.10). Invariance of the reparametrization Maurer-Cartan form (6.7) implies invariance of

$$
d \eta=d \alpha \wedge \omega
$$

and thus invariance of the one-form

$$
\zeta=d \alpha+\beta \omega
$$

in which $\beta$ is yet another pseudo-group parameter, representing the third reparametrization jet. Substituting (6.17) back into (6.16), we find

$$
\begin{aligned}
\boldsymbol{\mu}_{n+1} & =A^{-1} d \mathbf{a}_{n+1} \\
& =\left(A^{-1}\left[\mathbf{z}^{\prime \prime \prime}+\left(\alpha+(\log \lambda)^{\prime}\right) \mathbf{z}^{\prime \prime}+(\log \lambda)^{\prime \prime} \mathbf{z}^{\prime}\right]-\beta \mathbf{e}_{1}\right) \omega+\mathbf{e}_{1} \zeta .
\end{aligned}
$$

We are able to specify a normalization of the form

$$
\boldsymbol{\mu}_{n+1}=\left(K_{1} \omega+\zeta\right) \mathbf{e}_{1}+\omega \mathbf{e}_{2}
$$

for some $K_{1}$. Indeed, multiplying both sides of (6.18) by $A$,

$$
\mathbf{z}^{\prime \prime \prime}+\left(\alpha+(\log \lambda)^{\prime}\right) \mathbf{z}^{\prime \prime}+\left((\log \lambda)^{\prime \prime}-\beta\right) \mathbf{z}^{\prime}=K_{1} \mathbf{a}_{1}+\mathbf{a}_{2} .
$$

Taking the symplectic product of the latter identity with $\mathbf{a}_{1}=\mathbf{z}^{\prime}$, we find

$$
\left\langle\mathbf{z}^{\prime} ; \mathbf{z}^{\prime \prime \prime}\right\rangle+\left(\alpha+(\log \lambda)^{\prime}\right)\left\langle\mathbf{z}^{\prime} ; \mathbf{z}^{\prime \prime}\right\rangle=\alpha+(\log \lambda)^{\prime}=K_{1}\left\langle\mathbf{a}_{1} ; \mathbf{a}_{1}\right\rangle+\left\langle\mathbf{a}_{1} ; \mathbf{a}_{2}\right\rangle=0
$$


where the vanishing of

$$
\left\langle\mathbf{z}^{\prime} ; \mathbf{z}^{\prime \prime \prime}\right\rangle=0
$$

follows from differentiating (6.15). Therefore, the pseudo-group parameter must assume the value

$$
\alpha=-(\log \lambda)^{\prime}=-\frac{\langle\dot{\mathbf{z}} ; \dddot{\mathbf{z}}\rangle}{3\langle\dot{\mathbf{z}} ; \ddot{\mathbf{z}}\rangle^{4 / 3}} .
$$

Substituting back into (6.13), we find

$$
\mathbf{a}_{n+1}=\mathbf{z}^{\prime \prime}, \quad \text { and hence } \quad \eta=0, \quad \boldsymbol{\mu}_{1}=\omega \mathbf{e}_{n+1} .
$$

Moreover, substituting (6.20) into (6.17), results in the invariant one-form

$$
\zeta=\left((\log \lambda)^{\prime \prime}-\beta\right) \omega
$$

which can be normalized to 0 by setting

$$
\beta=(\log \lambda)^{\prime \prime}
$$

As a result, the normalization equation (6.19) reduces to

$$
\mathbf{z}^{\prime \prime \prime}=K_{1} \mathbf{a}_{1}+\mathbf{a}_{2},
$$

where

$$
K_{1}=-\left\langle\mathbf{a}_{n+1} ; \mathbf{z}^{\prime \prime \prime}\right\rangle=-\left\langle\mathbf{z}^{\prime \prime} ; \mathbf{z}^{\prime \prime \prime}\right\rangle
$$

is our first differential invariant, while

$$
\mathbf{a}_{2}=\mathbf{z}^{\prime \prime \prime}-K_{1} \mathbf{a}_{1}=\mathbf{z}^{\prime \prime \prime}+\left\langle\mathbf{z}^{\prime \prime} ; \mathbf{z}^{\prime \prime \prime}\right\rangle \mathbf{z}^{\prime} .
$$


Further, since $\zeta=0,(6.18)$ reduces to

$$
\boldsymbol{\mu}_{n+1}=\left(K_{1} \mathbf{e}_{1}+\mathbf{e}_{2}\right) \omega
$$

The next step is to normalize the Maurer-Cartan forms

$$
\boldsymbol{\mu}_{2}=A^{-1} d \mathbf{a}_{2}=\left\langle\mathbf{a}_{n+1} ; \mathbf{a}_{2}^{\prime}\right\rangle \mathbf{e}_{1}+\left\langle\mathbf{a}_{1} ; \mathbf{a}_{2}^{\prime}\right\rangle \mathbf{e}_{n+1}+H_{2} \mathbf{e}_{n+2}=H_{2} \mathbf{e}_{n+2},
$$

where, by direct computation using the fact that $\left\langle\mathbf{z}^{\prime} ; \mathbf{z}^{\prime \prime \prime \prime}\right\rangle=-\left\langle\mathbf{z}^{\prime \prime} ; \mathbf{z}^{\prime \prime \prime \prime}\right\rangle$,

$$
H_{2}=\left\langle\mathbf{a}_{2} ; \mathbf{a}_{2}^{\prime}\right\rangle=\left\langle\mathbf{z}^{\prime \prime} ; \mathbf{z}^{\prime \prime \prime \prime}\right\rangle-\left\langle\mathbf{z}^{\prime \prime} ; \mathbf{z}^{\prime \prime \prime}\right\rangle^{2}
$$

in agreement with (4.13). The first two coefficients in (6.26) vanish since

$$
\begin{aligned}
& \left\langle\mathbf{a}_{1} ; \mathbf{a}_{2}^{\prime}\right\rangle=-\left\langle\mathbf{a}_{1}^{\prime} ; \mathbf{a}_{2}\right\rangle=-\left\langle\mathbf{z}^{\prime \prime} ; \mathbf{z}^{\prime \prime \prime}-K_{1} \mathbf{z}^{\prime}\right\rangle=0, \\
& \left\langle\mathbf{a}_{n+1} ; \mathbf{a}_{2}^{\prime}\right\rangle \mathbf{e}_{1}=-\left\langle\mathbf{a}_{n+1}^{\prime} ; \mathbf{a}_{2}\right\rangle=-\left\langle\mathbf{z}^{\prime \prime \prime} ; \mathbf{z}^{\prime \prime \prime}-K_{1} \mathbf{z}^{\prime}\right\rangle=0 .
\end{aligned}
$$

Thus, as a consequence of the normalization (6.26),

$$
\mathbf{a}_{2}^{\prime}=H_{2} \mathbf{a}_{n+2}, \quad \text { whence } \quad \mathbf{a}_{n+2}=\frac{\mathbf{a}_{2}^{\prime}}{H_{2}}=\frac{\mathbf{z}^{\prime \prime \prime \prime}+\left\langle\mathbf{z}^{\prime \prime} ; \mathbf{z}^{\prime \prime \prime}\right\rangle \mathbf{z}^{\prime \prime}+\left\langle\mathbf{z}^{\prime \prime} ; \mathbf{z}^{\prime \prime \prime \prime}\right\rangle \mathbf{z}^{\prime}}{\left\langle\mathbf{z}^{\prime \prime} ; \mathbf{z}^{\prime \prime \prime \prime}\right\rangle-\left\langle\mathbf{z}^{\prime \prime} ; \mathbf{z}^{\prime \prime \prime}\right\rangle^{2}}
$$

provided our curve satisfies the additional nondegeneracy condition $H_{2} \neq 0$.

At this stage, we have normalized the columns $\mathbf{a}_{1}, \mathbf{a}_{n+1}, \mathbf{a}_{2}, \mathbf{a}_{n+2}$ of the matrix $A$. The rest of the computation produces the remaining columns of $A$ in the order $\mathbf{a}_{3}, \mathbf{a}_{n+3}, \mathbf{a}_{4} \ldots$ by recursively normalizing those components the components of the corresponding Maurer-Cartan forms $\mu_{n+2}, \mu_{3}, \mu_{n+3}, \ldots$ that continue to depend on group parameters. Let us implement the basic induction step to complete the moving frame computation. Suppose, for $k \geq 3$, we have already specified the columns $\mathbf{a}_{1}, \mathbf{a}_{n+1}, \mathbf{a}_{2}, \ldots \mathbf{a}_{k-1}, \mathbf{a}_{n+k-1}$, by normalizing the Maurer-Cartan forms $\boldsymbol{\nu}, \boldsymbol{\mu}_{1}, \boldsymbol{\mu}_{n+1}, \ldots, \boldsymbol{\mu}_{n+k-2}, \boldsymbol{\mu}_{k-1}$, in that order. To next 
normalize $\mathbf{a}_{k}$, we use the coefficients of $\boldsymbol{\mu}_{n+k-1}$, leading to

$$
\mathbf{a}_{n+k-1}^{\prime}=\sum_{i=1}^{n}\left[-\left\langle\mathbf{a}_{n+k-1} ; \mathbf{a}_{n+i}^{\prime}\right\rangle \mathbf{a}_{i}+\left\langle\mathbf{a}_{n+k-1} ; \mathbf{a}_{i}^{\prime}\right\rangle \mathbf{a}_{n+i}\right]
$$

where we make use of the identities

$$
\left\langle\mathbf{a}_{k} ; \mathbf{a}_{i}^{\prime}\right\rangle=\left\langle\mathbf{a}_{i} ; \mathbf{a}_{k}^{\prime}\right\rangle \quad \text { for any } \quad 1 \leq i, k \leq 2 n,
$$

resulting from differentiation of (6.1). Owing to the order of normalization, the coefficients of $\mathbf{e}_{1}, \ldots, \mathbf{e}_{k-1}, \mathbf{e}_{n+1}, \ldots, \mathbf{e}_{n+k-1}$ in $\boldsymbol{\mu}_{n+k-1}$ are already fixed by earlier normalizations. We normalize the coefficient of $\mathbf{e}_{k}$ to equal one, and all the remaining coefficients to zero, whereby

$$
\begin{array}{rlrl}
\left\langle\mathbf{a}_{n+k-1} ; \mathbf{a}_{n+k}^{\prime}\right\rangle & =1, & & \\
\left\langle\mathbf{a}_{n+k-1} ; \mathbf{a}_{n+i}^{\prime}\right\rangle & =0, & & i>k, \\
\left\langle\mathbf{a}_{n+k-1} ; \mathbf{a}_{i}^{\prime}\right\rangle & =0, & i \geq n+k .
\end{array}
$$

For this choice of normalization constants, equation (6.28) reduces to

$$
\mathbf{a}_{k}=\mathbf{a}_{n+k-1}^{\prime}+\sum_{i=1}^{k-1}\left[\left\langle\mathbf{a}_{n+k-1} ; \mathbf{a}_{n+i}^{\prime}\right\rangle \mathbf{a}_{i}-\left\langle\mathbf{a}_{n+k-1} ; \mathbf{a}_{i}^{\prime}\right\rangle \mathbf{a}_{n+i}\right]
$$

which serves to normalize $\mathbf{a}_{k}$.

Using (6.31), we next normalize $\mathbf{a}_{n+k}$ via the Maurer-Cartan form $\boldsymbol{\mu}_{k}$. The coefficients of $\mathbf{e}_{1}, \ldots, \mathbf{e}_{k-1}, \mathbf{e}_{n+1}, \ldots, \mathbf{e}_{n+k}$ have already been fixed by our earlier normalizations. We can safely normalize all the remaining coefficients to zero, and so

$$
\left\langle\mathbf{a}_{k} ; \mathbf{a}_{k}^{\prime}\right\rangle \mathbf{a}_{n+k}=\mathbf{a}_{k}^{\prime}+\sum_{i=1}^{k-1}\left[\left\langle\mathbf{a}_{k} ; \mathbf{a}_{n+i}^{\prime}\right\rangle \mathbf{a}_{i}-\left\langle\mathbf{a}_{k} ; \mathbf{a}_{i}^{\prime}\right\rangle \mathbf{a}_{n+i}\right] .
$$


Assuming the nondegeneracy conditions

$$
H_{k}=\left\langle\mathbf{a}_{k} ; \mathbf{a}_{k}^{\prime}\right\rangle \neq 0, \quad k=1, \ldots, n,
$$

we are able to solve equation (6.32) for $\mathbf{a}_{n+k}$ and thereby continue the inductive procedure.

The remarkable fact is that most of the coefficients in the inductive normalization equations (6.31), (6.32) are zero! An easy induction on $k$, sinilar to that in the proof of Theorem 1, proves our main result.

Theorem 2 The functions

$$
H_{k}=\left\langle\mathbf{a}_{k} ; \mathbf{a}_{k}^{\prime}\right\rangle \neq 0, \quad K_{k}=\left\langle\mathbf{a}_{n+k} ; \mathbf{a}_{n+k}^{\prime}\right\rangle, \quad k=1, \ldots, n,
$$

are the fundamental differential invariants, with

$$
H_{1}=\left\langle\mathbf{a}_{1} ; \mathbf{a}_{2}\right\rangle=1 .
$$

Furthermore,

$$
\left\langle\mathbf{a}_{n+k+1} ; \mathbf{a}_{n+k}^{\prime}\right\rangle=1, \quad k=1, \ldots, n-1,
$$

while all other symplectic products besides those in (6.34), (6.36) vanish:

$$
\left\langle\mathbf{a}_{i} ; \mathbf{a}_{j}^{\prime}\right\rangle=0 \quad \text { for } \quad 1 \leq i, j \leq 2 n .
$$


The moving frame normalizations of $(A, \mathbf{b}) \in G$ are given by

$$
\begin{aligned}
\mathbf{a}_{1}=\mathbf{z}^{\prime}, & \mathbf{a}_{k+1}=\mathbf{a}_{n+k}^{\prime}-K_{k} \mathbf{a}_{k}+\left(1-\delta_{k 1}\right) \mathbf{a}_{k-1}, & k=1, \ldots, n-1, \\
\mathbf{b}=\mathbf{z}, & \mathbf{a}_{n+k}=\frac{\mathbf{a}_{k}^{\prime}}{H_{k}}, & k=1, \ldots, n .
\end{aligned}
$$

Substituting the moving frame formula (6.37) back into (6.2) produces the normalized Maurer-Cartan forms

$$
\begin{aligned}
\nu & =\mathbf{e}_{1} d s, \\
\boldsymbol{\mu}_{k} & =H_{k} \mathbf{e}_{n+k} d s, \\
\boldsymbol{\mu}_{n+k} & =\left[\left(1-\delta_{k 1}\right) \mathbf{e}_{k-1}+K_{k} \mathbf{e}_{k}+\left(1-\delta_{k n}\right) \mathbf{e}_{k+1}\right] d s,
\end{aligned}
$$

where $d s=\omega$ is the symplectic arc length element.

Corollary 3 The moving frame $(A, \mathbf{b})$ given by (6.37) satisfies the structure equations

$$
d A=B A d s, \quad d \mathbf{b}=\mathbf{a}_{1} d s,
$$

where $B$ is as in (4.12).

We have thus recovered the same moving frame, structure equations and a complete system of generating differential invariants as before.

\section{Curves with Constant Symplectic Curvatures.}

Our purpose in this section is to briefly discuss symplectic regular curves with constant local symplectic invariants, in the case in which the ambient symplectic manifold is $M=\mathbb{R}^{4}$, endowed with the standard symplectic form (2.1). The four-dimensional case is the lowest-dimensional case of interest from a symplectic point of view since the case where the ambient space is $\mathbb{R}^{2}$ corresponds to the 
unimodular affine geometry of plane curves, where it is well known that the curves with constant invariant are precisely the conics, [10].

One way to proceed would be to use the Theorem of Cartan which we referred to in the Introduction, which implies that the curves with constant symplectic curvatures are precisely the orbits of the one-parameter subgroups of the affine symplectic group in four variables, [9]. This would require the determination of all such one parameter subgroups. We shall proceed more directly by integrating the Frenet equations in the case in which all the symplectic curvatures are constant. This means of course that we restrict our attention to curves which admit a symplectic Frenet frame. In the four-dimensional case, the Frenet equations of Proposition 3 take the form

$$
\frac{d}{d s}\left(\begin{array}{l}
\mathbf{a}_{1} \\
\mathbf{a}_{2} \\
\mathbf{a}_{3} \\
\mathbf{a}_{4}
\end{array}\right)=\left(\begin{array}{cccc}
0 & 0 & 1 & 0 \\
0 & 0 & 0 & H_{2} \\
K_{1} & 1 & 0 & 0 \\
1 & K_{2} & 0 & 0
\end{array}\right)\left(\begin{array}{l}
\mathbf{a}_{1} \\
\mathbf{a}_{2} \\
\mathbf{a}_{3} \\
\mathbf{a}_{4}
\end{array}\right) .
$$

We assume that the symplectic curvatures $H_{2}, K_{1}$ and $K_{2}$ to be constant. The eigenvalues of the Frenet matrix appearing in the right hand side of (7.39) are given by

$$
\begin{array}{ll}
\mu_{1}=\frac{1}{\sqrt{2}} \sqrt{\lambda_{1}+\sqrt{\lambda_{2}}}, & \mu_{2}=-\frac{1}{\sqrt{2}} \sqrt{\lambda_{1}+\sqrt{\lambda_{2}}} \\
\mu_{3}=\frac{1}{\sqrt{2}} \sqrt{\lambda_{1}-\sqrt{\lambda_{2}}}, & \mu_{4}=-\frac{1}{\sqrt{2}} \sqrt{\lambda_{1}-\sqrt{\lambda_{2}}}
\end{array}
$$

where

$$
\lambda_{1}=K_{2} H_{2}+K_{1}, \quad \lambda_{2}=\left(K_{2} H_{2}-K_{1}\right)^{2}+4 H_{2} .
$$

Note that

$$
\begin{gathered}
\mu_{1}+\mu_{2}=0, \quad \mu_{3}+\mu_{4}=0, \\
\mu_{1} \mu_{3}=\mu_{2} \mu_{4}=\frac{1}{2} \sqrt{\lambda_{1}^{2}-\lambda_{2}}=\sqrt{H_{2}\left(K_{2} K_{2}-1\right)} .
\end{gathered}
$$


¿From (7.40), it is easy to determine the dependence of the spectrum of the symplectic Frenet matrix on the symplectic curvatures of the curve. Indeed, the spectrum is restricted by virtue of the constraints (7.42). If $\lambda_{2}>0, \lambda_{1}+\sqrt{\lambda_{2}}>0$ and $\lambda_{1}-\sqrt{\lambda_{2}}>0$, then the eigenvalues $\mu_{1}, \mu_{2}, \mu_{3}$ and $\mu_{4}$ are real and distinct. If $\lambda_{2}>0, \lambda_{1}+\sqrt{\lambda_{2}}>0$ and $\lambda_{1}-\sqrt{\lambda_{2}}<0$, then $\mu_{1}$ and $\mu_{2}$ are distinct real eigenvalues, while $\mu_{3}$ and $\mu_{4}$ are distinct complex conjugate eigenvalues. Similarly, if $\lambda_{2}>0, \lambda_{1}+\sqrt{\lambda_{2}}<0$ and $\lambda_{1}-\sqrt{\lambda_{2}}>0$, then $\mu_{1}$ and $\mu_{2}$ are distinct complex conjugate eigenvalues, while $\mu_{3}$ and $\mu_{4}$ are distinct real eigenvalues. If $\lambda_{2}>0, \lambda_{1}+\sqrt{\lambda_{2}}<0$ and $\lambda_{1}-\sqrt{\lambda_{2}}<0$, then the eigenvalues $\mu_{1}, \mu_{2}$ and $\mu_{3}, \mu_{4}$ come in distinct complex conjugate pairs. The cases where $\lambda_{2}>0$ and $\lambda_{1} \pm \sqrt{\lambda_{2}}=0$ are treated similarly, leading to zero being an eigenvalue of multiplicity two. This occurs whenever $K_{1} K_{2}=1$ or $H_{2}=0$. If $\lambda_{2}<0$, then the eigenvalues $\mu_{1}, \mu_{2}$ and $\mu_{3}, \mu_{4}$ also come in distinct complex conjugate pairs. If $\lambda_{2}=0$, then $\mu_{1}\left(=\mu_{3}\right)$ is, depending on the sign of $\lambda_{1}$, a real or a pure imaginary eigenvalue of multiplicity two, and we have $\mu_{2}=-\mu_{1}$ and $\mu_{4}=-\mu_{3}$. Depending on which of the cases listed above corresponds to the spectrum of the symplectic Frenet matrix, we obtain curves of constant curvatures which are "symplectic helices" of Euclidean or hyperbolic type, or degenerations thereof.

Acknowledgements: The research of the first author was supported in part by NSERC grant RGPIN 105490-2004, the second author by NSF Grant DMS 05-05293, and the third author by CNPq Grant 306117/2006-1. 


\section{References}

[1] J.C. Álvarez Paiva and C.E. Durán, Geometric invariants of fanning curves, Adv. Appl. Math. 42 (2009), 290-212.

[2] I.M. Anderson, Introduction to the variational bicomplex, Contemp. Math. 132 (1992), 51-73.

[3] M.S. Barbosa and B. de Sousa, Differential invariants for symplectic Lie algebars realized by boson operators, J. Phys. A 37 (2004), 4797-4812.

[4] E. Calabi, P.J. Olver and A. Tannenbaum, Affine geometry, curve flows, and invariant numerical approximations, Adv. Math. 124 (1996), 154-196.

[5] É. Cartan, La théorie des groupes finis et continus et la géométrie différentielle traitées par la méthode du repère mobile, Gauthier-Villars, 1937.

[6] S.S. Chern and H.C. Wang, Differential geometry in symplectic space, Science Report Nat. Tsing Hua Univ. 4 (1947), 453-477; reprinted in S.S. Chern, Selected Papers, Vol. II, pp. 165-191.

[7] I. Ekeland and H. Hofer, Symplectic topology and Hamiltonian dynamics, Math. Z. 200 (1989), no. 3, 355-378.

[8] M. Fels and P.J. Olver, Moving coframes. I. A practical algorithm, Acta Appl. Math. 51 (1998), 161-213.

[9] M. Fels and P.J. Olver, Moving coframes. II. Regularization and theoretical foundations, Acta Appl. Math. 55 (1999), 127-208.

[10] H.W. Guggenheimer, Differential Geometry, McGraw-Hill, New York, 1963. 
[11] P.J. Olver, Moving frames and singularities of prolonged group actions, Selecta Math. 6 (2000), 41-77.

[12] P.J. Olver, Moving frames - in geometry, algebra, computer vision, and numerical analysis, in: Foundations of Computational Mathematics, R. DeVore, A. Iserles and E. Süli, eds., London Math. Soc. Lecture Note Series, vol. 284, Cambridge University Press, Cambridge, 2001, pp. 267-297.

[13] X. Xu, Differential invariants of classical groups, Duke Math. J. 94 (1998), $543-572$. 